\title{
A CASE OF "MASSIVE OSTEOLYSIS" OF THE FEMUR
}

\author{
J. N. Aston, London, England \\ From the Orthopaedic Department, St Bartholomew's Hospital, London, \\ and the Institute of Orthopaedics, the Royal National Orthopaedic Hospital, London
}

Very rarely part of the skeleton fades away for no apparent reason. Gorham and Stout (1955) named this condition " massive osteolysis" and reported two cases, and they collected twenty-two further cases from the literature.

In all, the outstanding histological feature has been an increase in the number and size of capillaries engorged with red blood cells. The term " haemangiomatosis" was suggested. The process can start in any bone. It begins as a localised erosion, and from this, slow centrifugal absorption of a large piece of the skeleton takes place. Eventually the process becomes arrested, but only after widespread destruction has occurred. The condition seems to be rare enough to justify the report of another case.

\section{CASE REPORT}

A girl of eighteen was first seen in October 1951, complaining of pain in the left thigh of gradual onset and six months' duration. The pain was not severe; it varied considerably, but

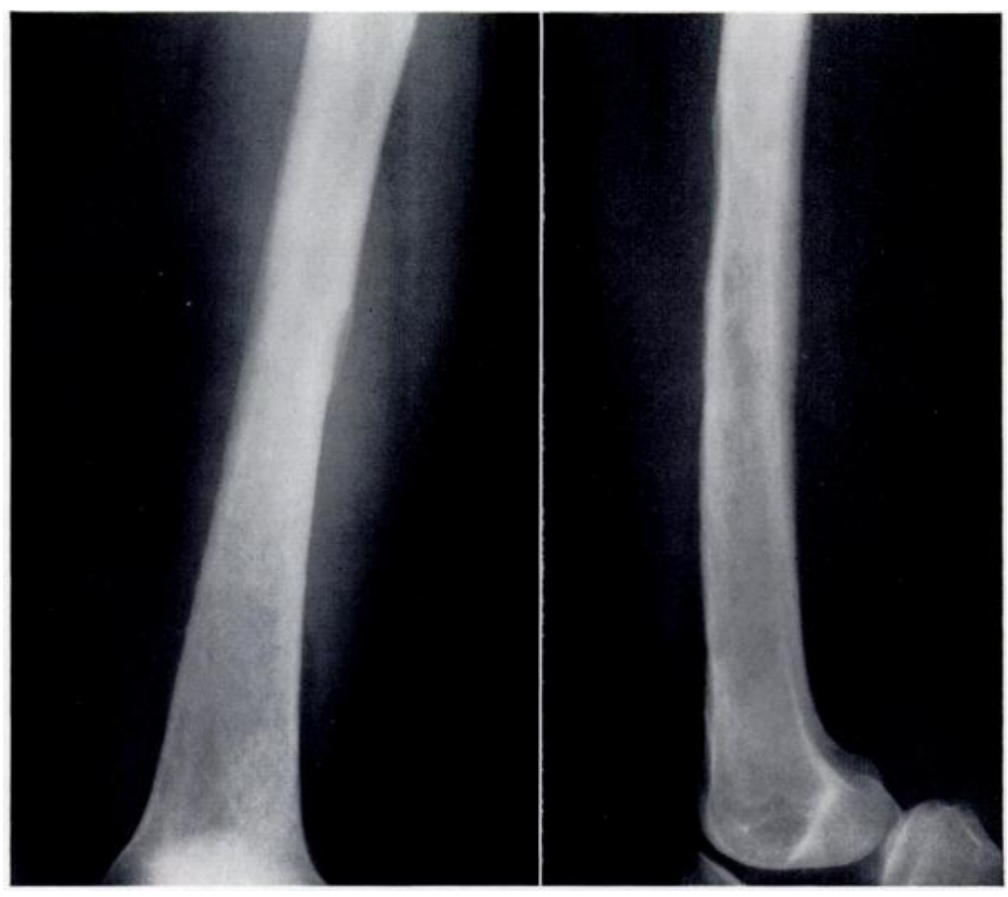

Fig. 1

Initial radiograph, showing early mid-femoral subperiosteal erosion.

was slowly becoming worse. Examination showed an inch of wasting of the left thigh and some deep tenderness at the junction of the middle and lowest thirds, with an impression of local thickening. Radiographs showed subperiosteal erosion of the femur (Fig. 1). 


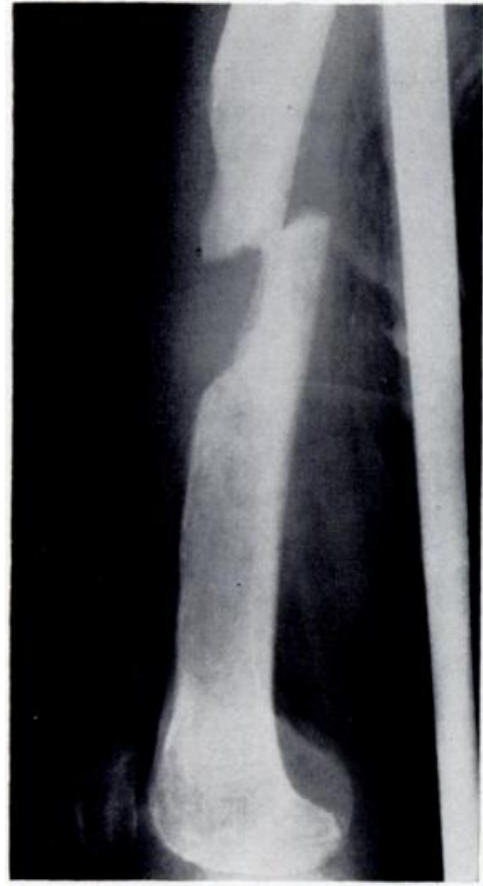

FIG. 2

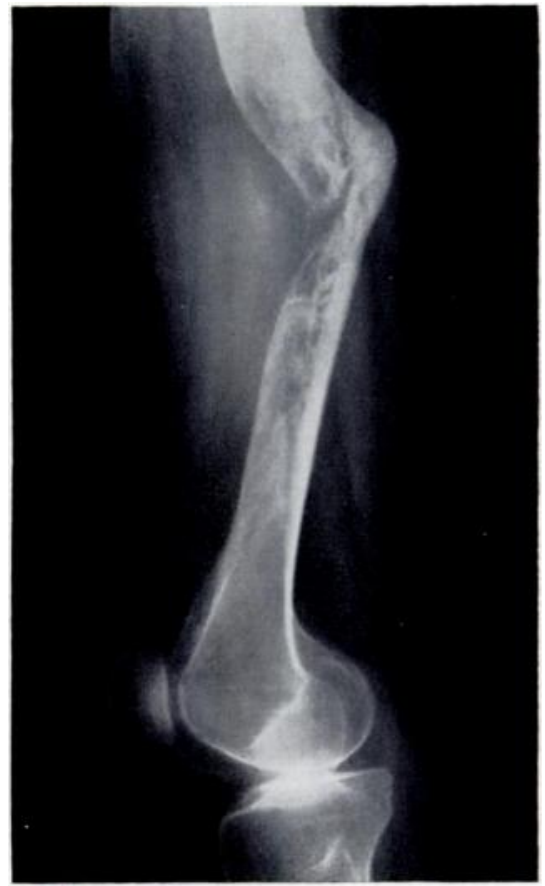

Fig. 3

Figure 2-Pathological fracture through site of biopsy; further erosion lower in the femoral shaft can be seen beginning. Figure 3-Sixteen months later the fracture has shown signs of union but further extensive erosion of the femoral shaft has caused collapse.

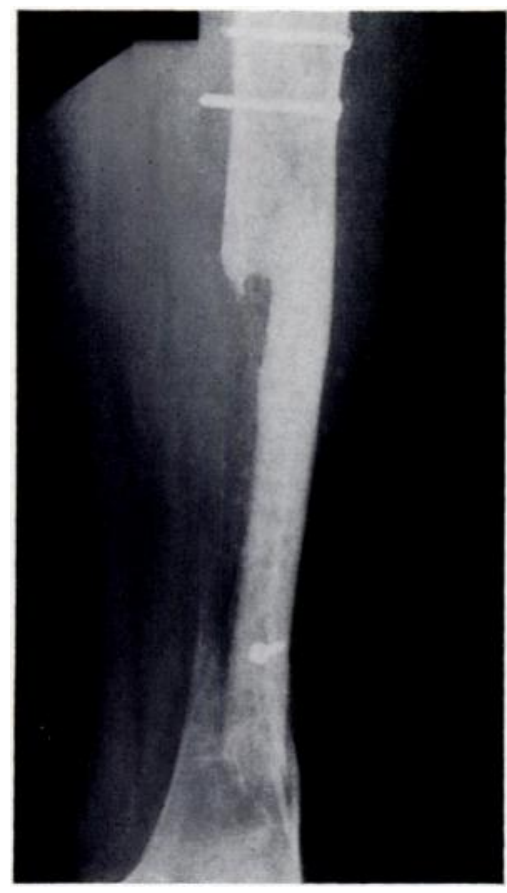

Fig. 4

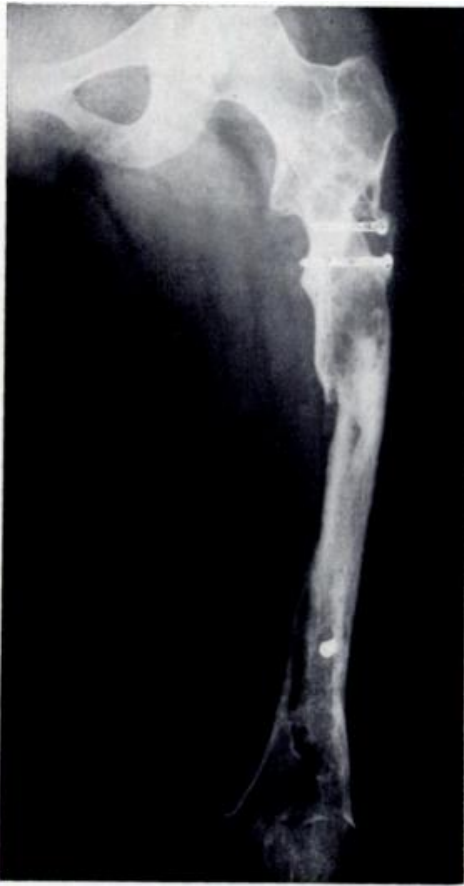

Fig. 5

Figure 4-Seventeen months after excision of the eroded area, showing incorporation of the graft. Figure 5-Radiograph immediately before the final operation, showing fresh erosion taking place in the femur, both above and below the graft.

VOL. 40 B, NO. 3, AUGUST 1958 
Biopsy was undertaken. The periosteum, though slightly thickened, stripped easily. The bone seemed rough and its cortex thin.

Histology (Dr R. J. R. Cureton)-Sectons were taken of the periosteum, muscle, bone and bone marrow. These showed a large number of prominent vessels, including arterioles, capillaries and vessels whose features were like those of an arteriovenous aneurysm. There was no question of any malignant change.

Progress-A pathological fracture occurred at the site of the biopsy (Fig. 2). This eventually showed signs of union, but, at the same time, erosion progressed to involve a large part of the mid-femoral shaft (Fig. 3). Amputation was offered, but declined.

In May 1953 (nineteen months after the biopsy) the eroded segment of bone was excised and the gap was bridged by a massive autogenous tibial graft. The graft slowly became

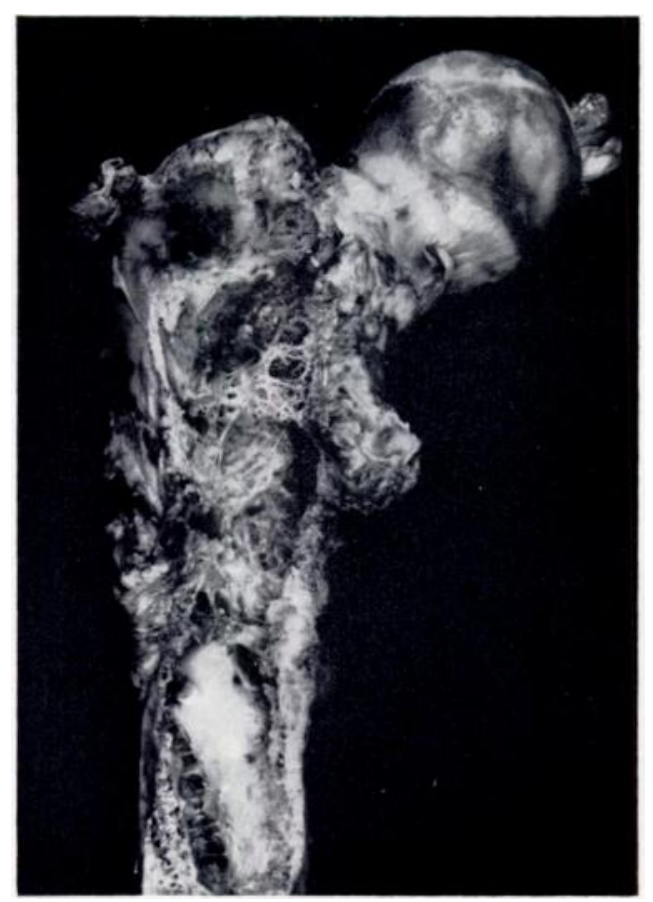

Fig. 6

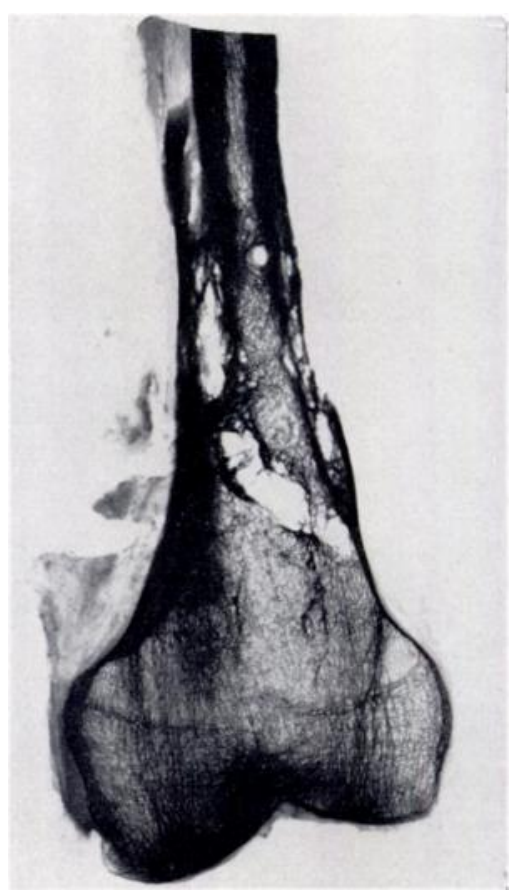

Fig. 7

Figure 6-Macroscopic appearance of the upper end of the femur. Figure 7-Slab radiograph of the lower end of the femur, showing erosion where the graft joins the bone, and also in the graft itself.

incorporated (Fig. 4), and after a further nineteen months the hip spica was removed and a weight-relieving caliper was fitted. This was discarded in July 1956. Two months later the patient slipped and sustained a fracture at the upper end of the graft. This fracture again slowly united, but further radiographs showed erosion taking place at both the upper and lower ends of the grafted area (Fig. 5). Finally, it was decided that the only real prospect of arresting the disease would be by total ablation of the femur.

Operation (May 1957) - Skin flaps were cut as for amputation through the thigh at the site of election, and the incision was extended upwards on the outer side of the thigh, finally splitting the gluteus maximus. The whole femur was then removed extraperiosteally (Seddon and Scales 1949) and the upper two-thirds replaced by an implant of cobalt-chrome-molybdenum 


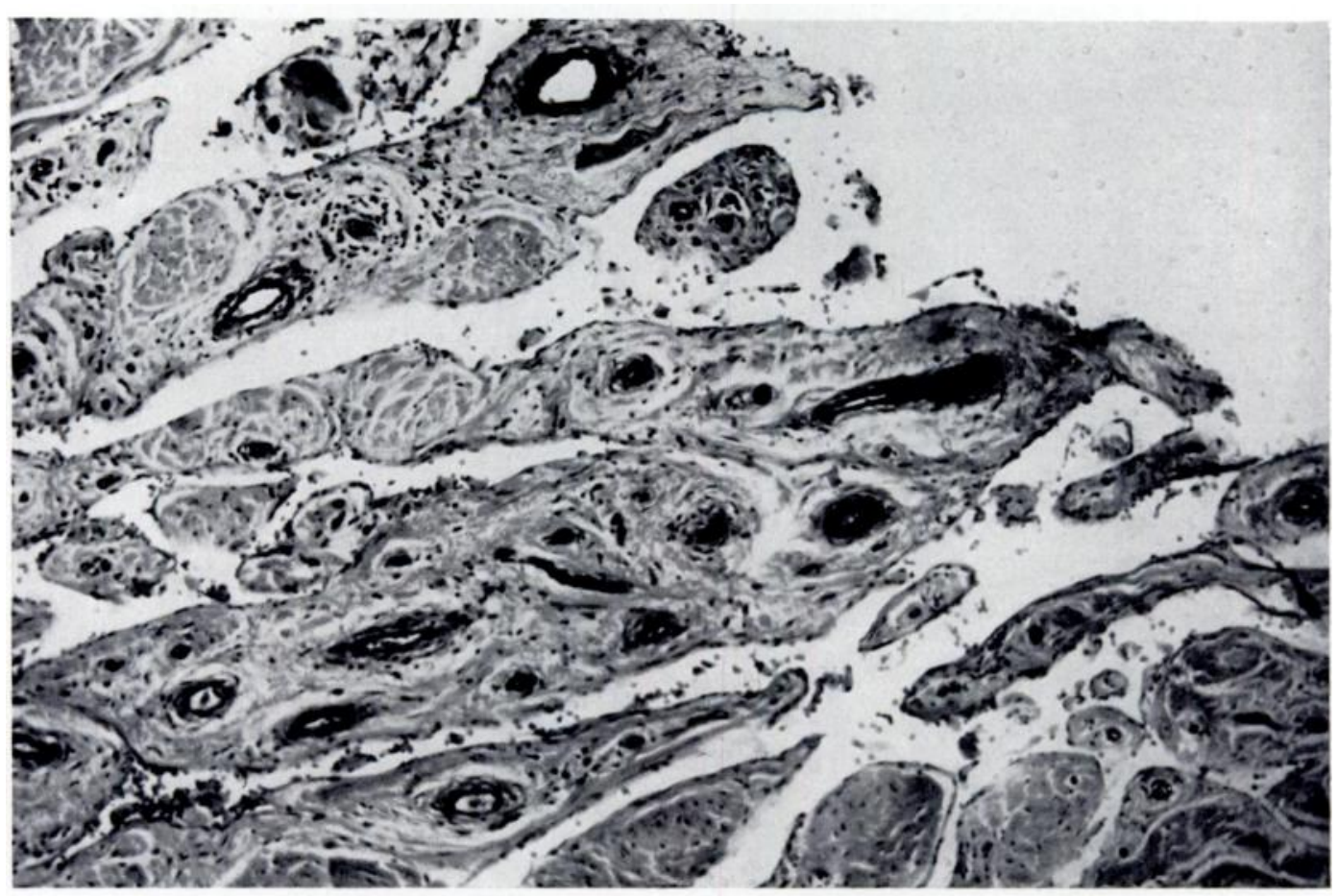

FIG. 8

Photomicrograph of part of the wall of the femoral lesion, showing a network of vascular fibrous tissue. $(\times 95$.

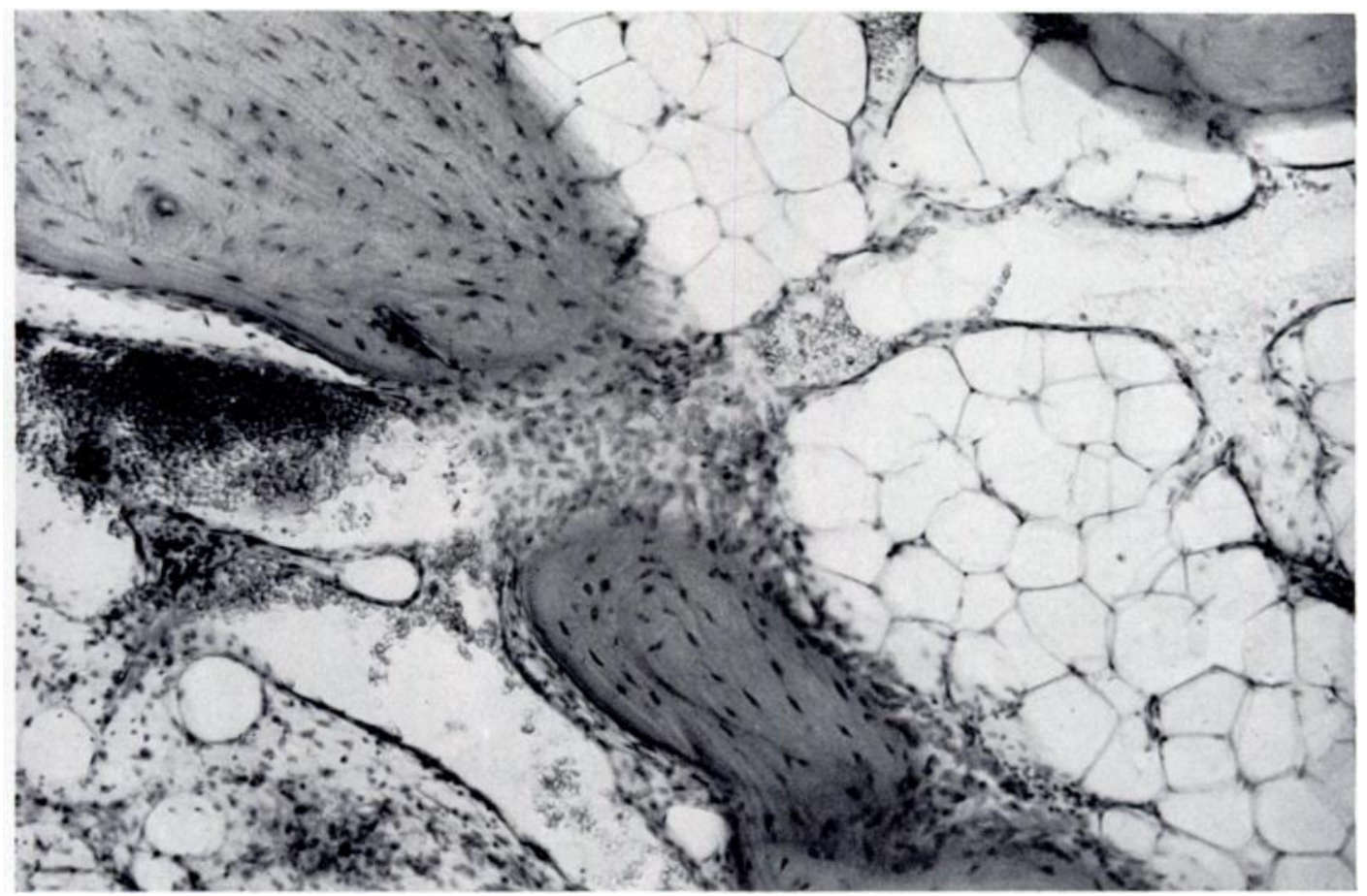

Fig. 9

Photomicrograph of the cancellous bone near the margin of the lesion, showing large dilated vascular spaces. ( $\times 95$.

vol. 40 B, NO. 3, AUGUSt 1958 
alloy to which the muscles were attached so that the patient could wear an above-knee artificial limb. She was fitted with this six months later.

The macroscopic appearance of the upper end of the femur is shown in Figure 6.

Histology-Dr H. A. Sissons reported that at the margins of the graft the upper and lower parts of the femur showed cystic spaces occupied and lined by cavernous or sinusoidal tissue (Fig. 6). This tissue appeared to be eroding the adjacent bone and, in the region of the femoral neck, showed some extension into the neighbouring soft tissue. The graft itself showed erosion by the vascular tissue. Some patches of acellular necrotic bone were present in it, but the bulk was replaced by newly formed cellular bone (Fig. 7). The characteristic vascular tissue present in the lesion of the femur suggested that it should be regarded as a type of angioma of bone (Figs. 8 and 9).

Most grateful thanks are due to $\mathrm{Mr} \mathrm{H}$. Jackson Burrows for permission to report this case, and for all the helpful advice and criticism he has given in the preparation of this report; to Dr R. J. R. Cureton and Dr H. A. Sissons for the histological details and to the Department of Medical Photography of the Institute of Orthopaedics for the illustrations.

The prosthesis, designed by Dr J. T. Scales of the Department of Biomechanics and Surgical Materials of the Institute of Orthopaedics, was cast in "Vinertia " by Messrs Trucast Ltd. and machined by Messrs Deloro Stellite.

\section{REFERENCES}

Gorham, L. W., and Stout, A. P. (1955): Massive Osteolysis (Acute Spontaneous Absorption of Bone, Phantom Bone, Disappearing Bone). Journal of Bone and Joint Surgery, 37-A, 985.

Seddon, H. J., and Scales, J. T. (1949): A Polythene Substitute for the Upper Two-thirds of the Shaft of the Femur. Lancet, ii, 795. 